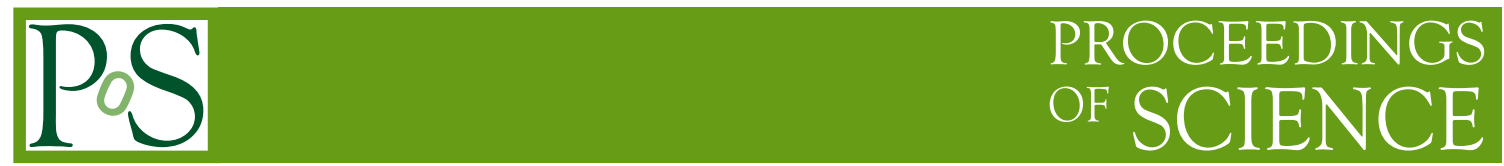

\title{
Summary of the Hadronic Final States Working Group
}

\author{
Vladimir M. Braun ${ }^{1}$, Daniel Britzger*2, Konstantinos Kousouris ${ }^{3}$ \\ ${ }^{1}$ Institut für Theoretische Physik, Universität Regensburg, D-93040 Regensburg, Germany \\ ${ }^{2}$ DESY, Notekstraße 85, 22607 Hamburg, Germany \\ ${ }^{3}$ CERN, CH-1211 Geneve 23, Switzerland \\ E-mail: vladimir.braundphysik.uni-regensburg.de, \\ daniel.britzgeradesy.de, konstantinos.kousouris@cern.ch
}

In this summary we give an overview on the experimental and theoretical topics which were presented during the sessions of the QCD and Hadronic Final State Working Group [1] at the DIS 2014 conference.

XXII. International Workshop on Deep-Inelastic Scattering and Related Subjects, 28 April - 2 May 2014

Warsaw, Poland

\footnotetext{
* Speaker.
} 


\section{Introduction}

The QCD and Hadronic Final State Working Group at the Deep Inelastic Scattering 2014 conference covered a wide range of experimental and theoretical topics. A combined session together with the Small-x, Diffraction and Vector Mesons Working Group further extended the range to the softer regime. This report gives a summary on the topics discussed in these sessions, where the experimental and theoretical contributions are mostly summarized separately.

The covered topics include among others the physics of jets, prompt photons and jets associated with weak bosons, the underlying event and double-parton scattering, particle production as well as tuning and validation of Monte Carlo event generators ${ }^{1}$.

\section{Experimental contributions}

Various experiments reported new and updated measurements to this working group. These are results from the ATLAS, CMS and LHCb collaborations at the LHC, H1 and ZEUS at HERA, as well as results from BaBar and NA48/62. The LHC experiments have reported on measurements in $p p$-collisions at a center-of-mass energy of $\sqrt{s}=0.9,7$ and $8 \mathrm{TeV}$, where the high luminosity of the $8 \mathrm{TeV}$ data enabled first measurements of QCD phenomena in association with rare processes, like for instance top quark pairs. The HERA experiments have shown new results with their final data precision and full data statistics and also more results are still expected in the future from these experiments.

\subsection{Jet production}

Studies of jet production are one of the benchmark processes to study our understanding of QCD, but are also very important analyses for a detailed understanding of the experimental setup. Recent measurements at the LHC provide precise data of QCD processes in an previously unexplored energy regime.

The ATLAS Collaboration has presented new results of cross sections for dijet production in $p p$ collisions at a center-of-mass energy of $\sqrt{s}=7 \mathrm{TeV}$ with an integrated luminosity of $4.5 \mathrm{fb}^{-1}[2$, 3]. Jets are reconstructed using the anti- $k_{t}$ algorithm with a jet radius parameter of 0.4 and 0.6 and cross sections are measured as function of the dijet mass up to masses of $5 \mathrm{TeV}$ for different regions of the rapidity separation of the two jets. The data are corrected for higher order electroweak and non-perturbative effects and compared to next-to-leading order perturbative QCD (pQCD) predictions obtained with various PDF sets. The data are in general well described by the theory predictions within the theoretical and experimental uncertainties. The high-mass region is potentially suited to further constrain the PDFs at high momentum fractions. The potential of these cross section measurements to constrain theoretical parameters, like PDFs, the strong coupling constant or physics beyond the Standard Model, is demonstrated by exploring contact-interactions (CIs) and a $95 \%$ confidence limit on the compositeness scale of CIs is set to 6.9 to $7.7 \mathrm{TeV}$, depending on the PDF set employed.

\footnotetext{
${ }^{1}$ In this brief summary not all references can be given and the reader is referred to the selected references and the references therein. This holds particularly for references on experimental data in theory talks or theoretical predictions for experimental results, like Monte Carlo event generators (MC), fixed order calculations or various analysis tools.
} 
ATLAS has further presented a measurement of jet-shape observables for jets from hadronically decaying b-quarks compared to light-quark jets in top-quark pair events $[4,5]$. It is observed that $b$-jets are wider than light jets, which is more pronounced at lower transverse momenta, and the jet shapes are well described by the studied MC models. It was discussed, that the difference of the b-jets compared to light jets is not sufficient to use these quantities for an efficient b-tagging. A further analysis of the measurement of the transverse momenta of $Z$-boson decaying into two b-quarks, which is a potential background in Higgs analyses, yields good agreement of data and NLO+PS predictions $[4,6]$.

The CMS Collaboration has presented several measurements of jet quantities in $p p$ collisions of 7 and $8 \mathrm{TeV}$ [7]. These are inclusive jet, dijet and 3-jet cross sections and measurements of event shapes, color-coherence effects and ratios of jets with different jet sizes.

The inclusive jet cross sections are now measured at $\sqrt{s}=7$ and $8 \mathrm{TeV}$ using an integrated luminosity of 5 and $11 \mathrm{fb}^{-1}$ of data, respectively $[8,10]$. The measurement at $8 \mathrm{TeV}$ is further extended to the region of low transverse momenta of the jets down to $p_{T}^{\text {jet }} \geq 21 \mathrm{GeV}$ using a lowpile up event sample with $5.8 \mathrm{pb}^{-1}$ [11]. The cross sections, corrected for non-perturbative effects, are described over the impressive range of 2 magnitudes well by predictions in next-to-leading order pQCD using nlojet++ [9] and various PDF sets, where data and theory predictions have uncertainties of comparable size.

Similar as the ATLAS Collaboration, CMS has also presented measurements of dijet cross sections in $p p$ collisions at $\sqrt{s}=7 \mathrm{TeV}$ using $5 \mathrm{fb}^{-1}$ of data [8]. The cross sections are presented as functions of the invariant mass of the two jets, up to $5 \mathrm{TeV}$, and of the maximum rapidity of the 2-jet system, $|y|_{\max }$. A good description by NLO predictions is observed within uncertainties over the whole kinematic range also using various PDF sets.

The measurement of 3-jet cross sections at $7 \mathrm{TeV}$ as function of the 3-jet mass and the maximum rapidity [12] are well described by pQCD NLO predictions within the uncertainties. The implication of these data in the extraction of the strong coupling constant $\alpha_{s}$ and in PDF fits is presented to this conference in the structure function working group [13].

The CMS Collaboration has further presented a measurement of the ratio of inclusive jet cross sections of two different jet sizes [14]. These are anti- $k_{t}$ cross section with jet radius parameter $R=0.5$ to jets with $R=0.7$. This quantity can be calculated directly using NLO predictions [15]. It was demonstrated that the ratio is sensitive to collinear radiation and non-perturbative effects are necessary to describe the data in $\mathrm{pQCD}$. Though, the $\mathrm{pQCD}$ predictions seem to overshoot the data. LO or NLO MC event generators matched to parton shower generators (Pythia, Herwig++ and POWHEG) give a reasonable description of the data.

Measurements of hadronic event shape variables, which are sensitive to the energy flow of the hadronic final state, are presented by CMS using data collected at $7 \mathrm{TeV}$ [16]. Observables like the thrust observable in the transverse plane is capable of separating between 2-jet and multi-jet topologies. The thrust observable is well described by the studied MC generators and tunes. Other observables, like the jet broadening or the jet mass event shape observables, are less well described by the studied MC generators.

CMS has further presented a study of color coherence effects, where effects from color interactions after the hard scatter during the fragmentation process are under investigation [17]. Color coherence effects are studied based on 3-jet events, where the two leading jets exhibit a back-to- 
back topology and a third parton was radiated which is supposed to lie in the plane defined by the emitting parton and the beam axis. The investigated observable $\beta$ is defined as the azimuthal angle of the third jet with respect to the second in the $\eta-\phi$ space. The data shows an enhancement due to the color coherence effects of events near $\beta \approx 0$ and a suppression in the transverse plane ( $\beta \approx \pi / 2$ ). The studied MC models do not provide a satisfactory description of this observable.

The measurements of hadronic event shapes and color coherence effects will be used for validation and further tuning of MC event generators.

\subsection{Productions of prompt photons}

The production of isolated photons in an hadronic environment, so-called prompt photons, is another important process to test the understanding of underlying QCD processes. Since prompt photons are unaffected by hadronisation they are a direct probe of the partonic hard process. Nonprompt photons, i.e. photons originating from decays of secondary particles such as $\pi^{0}$, are described using fragmentation functions. Precise measurements of prompt photons have the capability of constraining PDFs of the proton or the photon. The understanding of photon production at the LHC is of particular importance in view of the Higgs boson measurements, where the background for its photonic decay needs to be well modeled.

The ZEUS Collaboration has reported measurements on the production of isolated photons in photoproduction $[18,19,20]$, defined as $Q^{2}<1 \mathrm{GeV}$. Prompt photons have been measured inclusively and with an accompanying jet as function of the photon and for jet kinematic variables in the kinematic region of the photon of $-0.7<\eta^{\gamma}<0.9$ and with a transverse energy of $6<E_{T}^{\gamma}<15 \mathrm{GeV}$ [20]. The number of prompt photon events was determined by a fit to the energy-weighted width of the energy-cluster comprising the photon candidate which allows for a clear separation of background from decays of neutral mesons and isolated photons. The data are compared to NLO predictions including fragmentation terms [21] which give a good description of the data. The data are further compared to predictions using the $k_{T}$-factorization method (LMZ) [22] which describe the photon distributions well, but give a less good description at low $\eta^{\text {jet }}$ and for the resolved enhanced region. The uncertainties on the theory predictions obtained by scale variations are typically larger then the uncertainties of the data. Further detailed studies have been presented for photon plus jet cross sections for the direct-enhanced and the resolved-enhanced regions separately, where also observables which are sensitive to higher order radiation, like the rapidity separation of the jet and the photon, have been investigated [19]. Both theory predictions give mostly a good description of these data within large theoretical uncertainties. The LMZ-predictions have problems describing the rapidity distribution of the jet for the resolved-enhanced region. The double-differential cross sections presented are potentially valuable input to future photon PDF fits.

The ATLAS Collaboration has presented measurements of the production of isolated photons inclusively and with an accompanying jet $[23,24]$ using data with an integrated luminosity of $4.6 \mathrm{fb}^{-1}$ recorded in $p p$ collisions at $\sqrt{s}=7 \mathrm{TeV}$. Photons are measured in the kinematic region of $0<\left|\eta^{\gamma}\right|<2.37$ and with a transverse energy of $E_{T}^{\gamma}>100 \mathrm{GeV}$. Backgrounds from neutral meson decays are highly suppressed by application of 'tight ID' criteria based on the electromagnetic shower profiles and by requiring the photon to be isolated. The data are compared to NLO predictions using the JETPHOX program [25], which are lower than data for low $E_{T}^{\gamma}$ but in agreement within theoretical uncertainties. Predictions by MC generators Pythia and Herwig give a fair de- 
scription of the data. JETPHOX predictions are further obtained for different PDF sets. A study based on a pull distribution taking all experimental uncertainties into account reveals a tension for $E_{T}^{\gamma}<200 \mathrm{GeV}$, which is mostly independent on the applied PDF set. However, the theoretical uncertainties are often larger than the experimental ones and NNLO predictions are desired to fully exploit the high experimental precision.

Cross sections for photons in association with a jet have been also measured by the CMS Collaboration using data at $\sqrt{s}=7 \mathrm{TeV}[26,27]$. The transverse momentum of the photon is required within $40<p_{T}^{\gamma}<300 \mathrm{GeV}$ and of the leading jet of $p_{T}^{\text {jet }}>30 \mathrm{GeV}$. The cross sections are determined triple differentially in 8 different photon and leading jet $|\eta|$-configurations. The data are also well described by the JETPHOX NLO prediction over most of the kinematic regions except for cases of photons measured with large values of $|\eta|>2$.1. Predictions using SHERPA tend to be below the NLO predictions and provide a reasonable description over the whole kinematic range measured.

ATLAS has further studied the dynamics of cross sections of an isolated photon with a jet using $37 \mathrm{pb}^{-1}$ of data at $\sqrt{s}=7 \mathrm{TeV}[23,28]$. The angular distributions between the jet and the photon, $\left|\cos \theta^{\gamma j \mathrm{jet}}\right|$, are sensitive to the spin of the exchanged virtual particle and can such separate between photons radiated from direct-photon processes and originating from fragmentation processes. The photon is measured in the kinematic range of $0<\left|\eta^{\gamma}\right|<2.37$ and $E_{T}^{\gamma}>45 \mathrm{GeV}$. Jets are reconstructed using the anti- $k_{t}$ algorithm with $R=0.6$ and a transverse momentum of $p_{T}^{\text {jet }}>40 \mathrm{GeV}$ and $0<\left|\eta^{\text {jet }}\right|<2.37$. The data are well described by NLO predictions from JETPHOX. The shape of the $\left|\cos \theta^{\text {rjet }}\right|$ distribution is closer to that of the direct process than that of the fragmentation process.

\subsection{Jet production in association with weak bosons}

Studies of the production of vector bosons in association with jets feature small experimental uncertainties on the reconstruction of the vector boson and high precision $\mathrm{pQCD}$ prediction in NLO or ME+PS programs. These processes are important for modeling backgrounds for Higgs measurements or searches for physics beyond the Standard Model.

The productions of jets in association with a vector boson has been studied by the CMS Collaboration [26] for many final state configurations.

The production of $Z+$ jets [26, 29] and $W+$ jet cross sections [26, 30], in the $Z \rightarrow \ell \ell$ and $W \rightarrow \mu v$ channels, respectively, are measured inclusively for up to 6 accompanying jets with $p_{T}^{\text {jet }}>30 \mathrm{GeV}$ and pseudorapidities within $\left|\eta_{\text {jet }}<2.4\right|$. Differential cross sections are presented as a function of the transverse momentum and pseudorapidity for the four most energetic jets and as function of the $H_{T}$ variable for up to 4 jets. The data are normalized to the inclusive $W / Z$ production cross section and its predictions are obtained in NNLO precision by DYNNLO. The data shows good agreement with predictions from ME+PS event generators (Sherpa, MADGRAPH+Pythia) and with NLO predictions (Blackhat+Sherpa, POWHEG-BOX+Pythia), if these are available, within partially large experimental and theoretical uncertainties. The rapid evolving predictions in NLO for high jet multiplicities would motivate to measure even up to higher jet multiplicities.

CMS has further presented the measurement of the $Z+2$ jet final state cross sections in electroweak production using data of an integrated luminosity of $19.7 \mathrm{fb}^{-1}$ recorded at $\sqrt{s}=8 \mathrm{TeV}[26$, 31]. The mass of the dilepton system from the $Z$ decay is required to be $M_{\ell \ell}>50 \mathrm{GeV}$ and of the 
dijet mass to exceed $M_{j j}>120 \mathrm{GeV}$, with jets within $\left|\eta_{\text {jet }}\right|<5.0$. Two different methods are used for this analysis. Once a multi-variate analysis is performed, where the Z-boson is reconstructed only in the dimuon decay channel. As second analysis, the analysis is performed using dijet Fisher discriminant fits where the dimuon and dielectron decay channels of the $Z$-boson is investigated. Although both analysis are fairly compatible with each other, the combined result of the dimuon channel of the two analysis is well compatible with the theoretical predictions in NLO by VBFNLO.

CMS has further presented the measurement of rapidity distributions of events containing a $Z$ boson or a photon and one jet $[26,32,33]$. The measurements as function of the boson or jet rapidity are reasonably well described by the NLO predictions (MCFM for Z+jet and Owens for $\gamma+$ jet [34]) and by MC event generators (Sherpa and MADGRAPH). However, the predictions have some problems describing the rapidity separation and rapidity sum of the jet and the boson system.

Last but not least, the LHCb Collaboration has presented a measurement of the cross section for $Z$-boson production in association with a jet [35, 36]. The $Z$-boson is reconstructed via the decay into two muons. Jets are reconstructed using a particle flow algorithm taking tracking information and calorimeter deposits into account. The anti- $k_{t}$ algorithm with a jet size parameter of $R=0.5$ is employed for clustering to form jets with transverse momentum of $p_{T}^{\text {jet }}>10 \mathrm{GeV}$. Both, the Z-boson and the jet, are required to be within unique acceptance of the LHCb experiment of $2.0<\eta^{\mu \text {,jet }}<4.5$. The inclusive and differential cross sections are normalized to the inclusive $Z$ cross sections are are well described by POWHEG+Pythia predictions using different PDFs, as well as by NNLO predictions from FEWZ. The analysis presented is the first published analysis by the LHCb experiment involving the measurement of jets.

\subsection{Top pair events with additional radiation}

The ATLAS Collaboration has presented several studies on the associated production of a topquark pair and additional radiation [37]. These processes allow to test QCD at the scale of the top quark and provide important information for further tuning MC event generators. Events with top quark pairs are also irreducible backgrounds to Higgs analyses, e.g. in $t \bar{t} H$ or $H \rightarrow b \bar{b}$ final states, and searches for physics beyond the Standard Model, and reduced uncertainties on the modeling of top quark pair production mechanism is therefore crucial in particular for future LHC runs.

The modeling of additional radiation in top-quark pair events, reconstructed in the dilepton channel, was studied using gap fraction variables which are ratios of number of events without jet to number of events with an additional jet $[37,38,39]$. In the central rapidity region all studied MC event generators provide reasonable description of the variables, but the models have some problems in the forward region. A study of jet multiplicities in top pair events in the lepton+jets channel shows a reasonable description of the data by predictions using MC@NLO+Herwig, Alpgen+Herwig and Alpgen+Pythia within partially large experimental uncertainties, although the predictions differ significantly for large jet multiplicities [37, 38]

ATLAS has presented a measurement of the ratio of the fiducial cross section for the production of $t \bar{t}$ events with at least one heavy-flavor jet ( $b$ or $c$ ) to the one with a jet of any flavor, and the ratio was found to be in agreement with LO and NLO SM predictions [37, 40].

ATLAS has further presented a study of the transverse momentum of the $t \bar{t}, p_{T}^{t \bar{t}}$ pair, which is sensitive to additional higher order radiation $[37,41]$. The cross sections presented are in agreement 
with the predictions by Alpgen+Herwig, POWHEG+Herwig and MC@NLO+Herwig, while the latter one is closest to the data.

The measurement of top-quark pairs in association with a photon yields a significant signal consistent with the theoretical expectations [42]. The measurement of top-quark pairs in association with a vector boson lacks in statistics and only one event is observed in the studied data sample of $4.7 \mathrm{fb}^{-1}$ recorded at $\sqrt{s}=7 \mathrm{TeV}[43]$.

\subsection{Particle production}

The LHCb Collaboration has presented a measurement of charged particle production in the forward region of $2.0<\eta<4.8$ [44]. The measurement was performed using a minimum-bias data sample of $p p$ collisions at a centre-of-mass energy of $7 \mathrm{TeV}$ collected in the year 2010, where pile-up occurred in only $4 \%$ of the events. Charged particles are measured with momenta of $p>2 \mathrm{GeV}$ and transverse momenta greater than $p_{T}>0.2 \mathrm{GeV}$. Measurements of charged particle multiplicities and densities are compared to predictions from the Pythia and Herwig++ MC event generators with different tunes. For low values of $p_{T}$ of $0.2<p_{T}<0.3 \mathrm{GeV}$ the charged particle multiplicity is overestimated by Herwig++ and underestimated by Pythia. For high values of $p_{T}$, which is more sensitive to the hard process, the MC generators agree well with the data. The total multiplicity is underestimated by both generators. The data are going to be released as a RIVET analysis, which enables to compare the data to further models of particle production and to provide input to future generator tunes.

\subsection{Underlying event measurements and double-parton scattering}

Activity resulting from collisions besides the hard interaction plays an important role at hadronhadron collisions at high energies. The so-called underlying event (UE) is defined as all the activity in an event resulting from the collision besides the hard interaction, and is a product from initial and final state radiation, multi parton interactions (MPI) and the beam remnants. The observed UE activity is to some extent independent of the hard scattering scale. Since the UE is an irreducible background to many analyses and cannot be calculated in PQCD it needs to be well modeled using $\mathrm{MC}$ event generators, where many parameters of these models have to be tuned to describe the data.

The ATLAS $[45,46]$ and the CMS collaborations $[47,48,49,50,51]$ have reported on measurements of the UE event activity. Data are recorded during low-pile up runs in $p p$-collisions at $\sqrt{s}=7 \mathrm{TeV}$, and also at $\sqrt{s}=0.9 \mathrm{TeV}$ collisions for CMS. Charged and neutral particle properties (such as particle multipicites, particle densities, momenta sums, averaged momenta, etc...) are measured for different regions in the transverse plane defined by the hardest object in the event. In general, the data in the central region are well described by the MC event generators and tunes studied [45, 46, 47, 48], where only pre-LHC tunes have little problems. However, more detailed studies reveal some problems of the MC generators: ATLAS has studied the UE activity in the full acceptance up to $|\eta|<4.9$, and it was observed, that it is difficult for the MC models to describe the full $\eta$-range. CMS on the other hand has studied the strange particle production in underlying events in $p p$-collisions at $\sqrt{s}=7 \mathrm{TeV}$ and it was shown that all predictions (Pythia6 with the D6T, Z1 and Z2* tunes and Pythia8 with Tune 1) show significant differences to the measurements [47, 49]. 
A study on the UE was performed by CMS using a different approach $[47,50]$, where only tracks (so-called 'soft tracks') which are not clustered within an anti- $k_{t}$ jet with distance parameter $R=0.5$ and $p_{T}^{\text {jet }}>5 \mathrm{GeV}$, are the constituents of the UE. The jet-radius was chosen such that the momentum spectrum of the UE tracks does not change. The Pythia6, Pythia8 and Herwig++ predictions studied provide only partly a satisfactory description of the data and for instance the highest multiplicities are not described by the models. The study demonstrated also the importance of the modeling of MPI.

The study of the UE was also performed by CMS for $t \bar{t}$ events based on data of $19.7 \mathrm{fb}^{-1}$ recorded at $\sqrt{s}=8 \mathrm{TeV}$, where pile-up tracks have been removed using event vertex information $[47,51]$. The data are fairly well described by the Madgraph+Pythia6 predictions using the $\mathrm{Z} 2 *$ tune.

The measurements of UE are used to obtain and validate new sets of parameters for modeling the UE with the MC models ('tunes') which will be used in future LHC runs [52, 53]. The new tunes are used to calculate the effective cross section, $\sigma_{\text {eff }}$, which characterizes the effective transverse area of hard partonic interactions between the colliding protons to values of $\sigma_{\text {eff }}=28$ to $30 \mathrm{mb}$. The effective cross section is also extracted from measurements which are particularly sensitive to double-parton scattering (DPS). CMS has reported on measurements of $W+2$ jet cross sections $[54,55]$ and 4 -jet cross sections $[52,56]$. The extraction of $\sigma_{\text {eff }}$ from $\mathrm{MC}$ tunes to these cross section measurements yield values of $\sigma_{\text {eff }}=19$ to $22 \mathrm{mb}$, and of $\sigma_{\text {eff }}=$ $20.7 \pm 0.8$ (stat.) \pm 6.6 (syst.) $\mathrm{mb}$ from a template fit to the $W+2$ jet cross sections. This difference of the $\sigma_{\text {eff }}$ reveals the difficulties in the description of softer and harder MPI.

Effects of DPS have been presented by ATLAS in the study of $W+$ prompt $-J / \psi$ cross sections, which have been observed with a significance of 5.2 $\sigma[57,58]$. However, the ratio of the fiducial cross section to the inclusive $W^{ \pm}$cross section suggests that single parton scattering is the dominant contribution to this cross section at low $P_{T}^{J / \psi}$.

The LHCb Collaboration has presented a study of charmed meson production together with a $Z$-boson in the forward region where contributions from double-parton scattering may become significant $[35,59]$. A first observation of this process has been made and future analyses with increased statistics will be capable of disentangling the contributions from single and double-parton scattering.

The size of DPS effects has been investigated theoretically by A. Szczurek for double charmed meson cross sections [60] and compared to LHCb data. It was demonstrated that neutral charmed meson pairs with large rapidity separation, and hence produced predominantly in the forward region, are an ideal process to study DPS effects. The speaker has further presented new calculations for single $D$-meson production using the $k_{t}$ factorization approach [60]. Similar as predictions in collinear NLO, these give slightly too small cross section compared to recent data, and the speaker suggested that contributions from many small subleading contributions are yet missing for this process.

\subsection{Measurement of the transverse momentum of a Z-boson}

The ATLAS Collaboration has presented a measurement of the transverse momentum spectrum of a $Z / \gamma^{*}$-boson in $p p$ collisions with a centre-of-mass energy of $\sqrt{s}=7 \mathrm{TeV}[61,62]$ in the $Z / \gamma^{*} \rightarrow \mu^{+} \mu^{-}$and $Z / \gamma^{*} \rightarrow e+e^{-}$channels. Normalized differential cross sections as a function of 
the transverse momentum of the boson $p_{T}^{Z}$ in the range of $0<p_{T}^{Z}<800 \mathrm{GeV}$ and $0<\left|\eta_{Z}\right|<2.4$ are compared to pQCD (FEWZ, DYNNLO) and resummed predictions (RESBOS) as well as to predictions from MC event generators (Pythia8, POWHEG8+Pythia, Sherpa, MC@NLO and Alpgen) with different levels of agreement. Measurements of $p_{T}^{Z}$ and the $\phi_{\eta}^{*}$-observable [63] were further used to tune the parton shower model parameters of the Pythia8 and POWHEG+Pythia8 generators to obtain an agreement of $2 \%$ for $p_{T}^{Z}<50 \mathrm{GeV}$.

\subsection{Search for QCD instantons}

Instantons are an intrinsic part of QCD as realizations of non-perturbative fluctuations of the gluon field and can be interpreted as tunneling transitions between topologically non-equivalent vacua. An experimental observation of instanton-induced processes would constitute a discovery of a basic non-perturbative QCD phenomenon.

The H1 Collaboration has presented a search for a signal of a QCD instanton in DIS at HERA in the kinematic region of $150<Q^{2}<15000 \mathrm{GeV}^{2}$ and an inelasticity of $0.2<y<0.7$ [64, 65]. The instanton signal is characterized by a "fire-ball" like topology with large particle multiplicities and is modeled using the QCDINS MC generator [66]. The expected signal is extracted using a probability density estimator (PDERS) with topological variables defined in the instanton restframe as input. The data are found to be consistent with the background also in the instanton enhanced region and no evidence for a QCD instanton is found. An upper limit of $1.6 \mathrm{pb}$ for the instanton cross section is extracted at a confidence limit of $95 \%$ in comparison with a cross section predicted by QCDINS of $10 \mathrm{pb}$.

\subsection{Feynman Scaling in Photon and Neutron Production}

The H1 Collaboration has presented measurements of normalized cross sections for the production of photons and neutrons at very small angles w.r.t. the proton beam direction in deepinelastic ep scattering $[67,68]$. This measurement provides new information to improve the understanding of proton fragmentation. The data are presented as a function of the Feynman variable $x_{F}$ and of the center-of-mass energy of the virtual photon-proton system, $W$, and are compared to predictions obtained using the ep DIS MC event generators LEPTO, CDM (DJANGOH+ARIADNE) and RAPGAP- $\pi$. The models overestimate the rate of photons by 30 to $70 \%$ with large differences of the shapes between the models. The cross sections for forward neutrons are well described. The data are further compared to predictions of models which are commonly used for the simulation of cosmic ray cascades (SIBYLL 2.1, QGSJET 01, QGSJET II-04 and EPOS LHC), but none of the models describes the photon and neutron data simultaneously well. The data will be useful input for tuning and further improving MC model simulations of collider and cosmic ray data.

\subsection{Light meson production and rare meson decays}

The BaBar Collaboration has reported on measurements of light meson production cross sections, $\pi^{+} \pi^{-}(\gamma)$ and $K^{+} K^{-}(\gamma)$, in $e^{+} e^{-}$collisions at $\operatorname{SLAC}(\sqrt{s}=10.58 \mathrm{GeV})[69,70,71,72]$. The cross sections are measured by identifying events with a photon radiated in the initial state with $E_{\gamma}^{*}>3 \mathrm{GeV}$ which effectively lowers the center-of-mass energy of the hard process from threshold to up to $\sqrt{s^{\prime}}=3$ to $5 \mathrm{GeV}$. A comparison of the fitted cross sections of $e^{+} e^{-} \rightarrow \pi^{+} \pi^{-}(\gamma)$ shows 
some tension compared to measurements by KLOE, which needs some investigation of the systematics of the two experiments. The computation of the hadronic vacuum polarization contribution to $g-2$ (HVP) from the $\pi^{+} \pi^{-}(\gamma)$ data, $a_{\mu}^{\pi \pi(\gamma), \mathrm{LO}}$, shows a tension to previous extractions from the same process, but is in agreement with data from $\tau$-decays and is getting closer to the SM prediction. The precision of $a_{\mu}^{K^{+} K^{-}(\gamma)}$ from $K^{+} K^{-}(\gamma)$ cross sections improves by a factor of 2.6 compared to the previously best value. The Babar ISR-program covers a large variety of final state measurements and further results are to be expected.

The NA48/2 Collaboration has reported on the measurement of the charged kaon rare decays $K_{e 4}, K^{ \pm} \rightarrow \pi^{+} \pi^{-} e^{ \pm} v\left(K_{e 4}(+)\right)$ and $K^{ \pm} \rightarrow \pi^{0} \pi^{0} e^{ \pm} v\left(K_{e 4}(00)\right)$, and the measured branching ratios are much more precise than the previously known values [73, 74, 75].

The rare decay $K^{ \pm} \rightarrow \pi^{ \pm} \gamma \gamma$ has been studied at the NA48/2 beam line at the CERN-SPS by the NA48/2 and the NA62 Collaborations [76, 77, 78]. The measurements of the two collaborations are combined and the $K_{\pi \gamma \gamma}$ decay rate is extracted in a model-independent way as well as using Chiral Perturbation Theory (ChPT) and determining its free parameters $\hat{c}_{4}$ and $\hat{c}_{6}$. The results are in agreement with previous measurements and now overshoot the theoretical precision.

\section{Theoretical summary}

\subsection{Fragmentation functions}

H. Paukkunen [81] discussed the inclusive high-pT charged particle production in protonproton and proton-antiproton collisions with a special emphasis on the recent LHC and Tevatron measurements. The NLO calculations for the inclusive charged hadron prodution appear to significantly overshoot the CMS and ALICE data, that calls for a systematic study to chart the different sources of uncertainties and identify the causes of the mismatch. It appears that for the particle transverse momenta below $p_{T}=10 \mathrm{GeV}$ the scale uncertainty remains too large and prohibits any quantitative conclusions. In this region there is also evidence for a non-perturbative excess of baryons. On the other hand, for $p_{T}>10 \mathrm{GeV}$ the NLO calculation seems to sufficiently robust and the observed deviations can only be attributed to poorly known parton-to-hadron fragmentation functions: none of the existing sets can describe the data. Moreover, the discrepancy increases with the c.m. energy. It is suggested that the problem appears mainly from the presently too hard gluon-to-hadron fragmentation functions that have to be reanalyzed.

\subsection{High energy jet production}

Jet production at very high energies accessible at LHC calls for using various theory methods to resum energy logarithms that go back to the classical BFKL technique. In particular the study of the production of two forward jets with a large interval of rapidity was proposed by Mueller and Navalet as a possible test of the high energy QCD dynamics. B. Ducloue presented in his talk [80] the very recent results where this process was analyzed, for the first time, within a complete next-toleading logarithm framework supplemented by the use of the Brodsky-Lepage-Mackenzie (BLM) procedure extended to the perturbative Regge dynamics to find the optimal renormalization scale. The authors show that the agreement with CMS data at $7 \mathrm{TeV}$ is greatly improved by using the BLM scale fixing procedure and also the energy-momentum conservation is significantly improved 
by using the NLO jet vertex. One obtains a clear difference between NLO fixed-order and NLL BFKL predictions for azimuthal correlations between dijets, not affected by BLM.

Azimuthal decorelations in production of forward and central jets in $p p$ and $p P b$ collisions were also discussed by K. Kutak [84]. He presented the corresponding results obtained in a somewhat different approach, high energy factorization going back to the classical work by Ciafaloni, Catani and Hautmann and making use of gluon density obtained from the BK equation extended to account for some higher order corrections. The emphasis in this study is on combining different elements in this large program in a systematic way.

A competitative mechanism for the production of Mueller-Navalet jets is the double parton scattering (DPS) which is much less understood. R. Maciula presented in his talk [79] the study of DPS using a factorized ansatz in the LO collinear approximation which leads to a reasonably good description of the recent CMS and ATLAS data on inclusive jet production. Relative contribution of DPS is shown to grow at large rapidity distance between jets, consistent with previous studies of DPS effects in open and hidden charm production. A detailed study was done for the LHC energy and a broad jet transverse momenta range.

M. Jack in his talk [82] presented recent developments in the calculation of multi-jet events in multi-Regge kinematics in the framework of the High Energy Jets (HEJ) formalism based on Fadin-Kuraev-Lipatov effective vertex for real gluon emission. The new results presented at this conference include predictions for Higgs+jets and Z+jets production, that is achieved by including diagrams with one "unordered" emission in rapidity. Comparisons with experimental data and other Monte Carlo packages were presented that illustrate the differences and advantages of the all-order predictions by other methods from all-order predictions in HEJ.

\subsection{QCD Studies in the LHC high-luminosity phase}

In the forthcoming high-luminosity phase at the LHC, many of the most interesting measurements for precision QCD studies are hampered by conditions of large pileup, particularly at not very high transverse momenta. Finding observables that may be accessed in this environment presents a nontrivial problem. It was adressed in his talk by H. Van Haevermaet [83], who argued that measuring ratios of color-singlet currents via Higgs boson and Drell-Yan production is possible in the LHC high-luminosity runs and would provide new perspectives for the studies of gluon fusion processes, with a unique sensitivity to the gluon parton distribution. H. Van Haevermaet presented results of Monte Carlo calculations for a few specific examples. This is a large ongoing program which will be continued.

\section{$3.4 J / \Psi$ photoproduction}

A. Goritschnig [85] spoke of the photoproduction of $J / \Psi$ mesons off a proton in the kinematical regime of large energies and scattering angles within the framework of perturbative QCD. In these reactions the heavy charm-quark mass provides the relevant hard scale. The authors consider the standard, so-called hard-rescattering mechanism for the $J / \Psi$ where the large transfered momentum is redistributed by hard gluons coupling to the valence quarks. The authors observe that photoproduction at large $|t|$ is dominated by a specific subclass class of Feynman diagrams where the photon couples to one of the valence quarks of the proton before fluctuating in the $c \bar{c}$ 
pair. Predictions for the $\gamma p \rightarrow J / \Psi p$ cross section for the simplest models of hadron distribution amplitudes are given and compared, when possible, to the experimental data.

\subsection{Associated quarkonium production}

J.-P. Lansberg [86] presented a review of theoretical issues that arise in the calculations of heavy quarkonium production at high energies, with the emphasis on new opportunities in the LHC era. This is an old subject that is still full of controversies, e.g. there is up to now no final agreement on the relative importance of color-singlet and color-octet contributions. This situaltion is fuelling for the study of new observables which can be more discriminant to specific effects. The authors consider a number of such possibilities. They argue that back-to-back production of quarkonium + isolated photon provides a unique way to access the transverse dynamics and polarization of gluons inside the proton, encoded in transverse momentum dependent parton distributions (TMDs), whereas final states such as quarkonium $+W / Z$ can be of great help to discriminate between different quarkonium production mechanisms and also can shed light on double-parton scattering contributions. The quarkonium-pair production presents a yet another example and a potentially rich source of information which still has to be harvested.

\subsection{Exclusive central diffractive meson production}

Last but not least, P. Lebiedowicz gave a talk [87] on the exclusive central diffractive production of mesons in proton-proton collisions in a model based on a kind of reggeon field theory. In this model the amplitudes are formulated in terms of effective vertices and propagators for the exchanged pomeron and reggeons. Different pomeron-pomeron-meson tensorial (vectorial) coupling structures are possible in general. In most cases two lowest orbital angular momentum - spin couplings are necessary to describe experimental differential distributions. The authors specifically discuss the differences between results of the "tensorial pomeron" and "vectorial pomeron" models. The theoretical results are compared with the WA102, H1 and ZEUS experimental data, in order to determine the model parameters. It is shown that high-energy central exclusive production, in particular of pseudoscalar mesons, can provide crucial information on the spin structure of the soft pomeron, in particular for the pomeron coupling to particles with spin-one such as $\rho_{0}$ mesons.

\section{Conclusion}

The QCD and Hadronic Final State Working Group was very lively with a wide variety of new results mostly from the LHC experiments, where several specific LHC-related issues like multijets, double-parton scattering, underlying event, etc. are coming significantly forward. The breadth of the contributions proves that the topic of final states is very much alive. Promising outlooks on future LHC results at 13 and $14 \mathrm{TeV}$ with high luminosities and the exploration of new observables in previously unexplored energy regimes pointed out the future intensity and the continuous attraction of the field.

\section{References}

[1] V. Braun, D. Britzger, K. Kousouris, WG4 Highlights: QCD and Hadronic Final States, http://indico.cern.ch/event/258017/session/0/contribution/59 
[2] G. Vardanyan, Measurement of the jet production cross section at $7 \mathrm{TeV}$, https://indico.cern.ch/event/258017/session/4/contribution/111

[3] G. Aad et al. [ATALS Collaboration], Measurement of dijet cross sections in pp collisions at $7 \mathrm{TeV}$ centre-of-mass energy using the ATLAS detector, JHEP 1405 (2014) 059 [arXiv:1312.3524]

[4] R. Chislett, Jet properties of hadronically-decaying massive particles, https://indico.cern.ch/event/258017/session/4/contribution/113

[5] G. Aad et al. [ATLAS Collaboration]Eur. Phys. J. C 73 (2013) 2676

[6] G. Aad [ATLAS Collaboration], Measurement of the cross section of high transverse momentum $p T$ production in proton-proton collisions at sqrts $=8 \mathrm{TeV}$ with the ATLAS Detector, CERN-PH-EP-2014-064 (2014) [arXiv:1404:7042]

[7] P. Kokkas, Jet production measurements at CMS, https://indico.cern.ch/event/258017/session/4/contribution/43

[8] S. Chatrchyan et al. [CMS Collaboration], Measurements of differential jet cross sections in proton-proton collisions at sqrts=7 TeV with the CMS detector, Phys. Rev. D 87 (2013) 112002 [arXiv:1212.6660]

[9] Z. Nagy and Z. Trocsanyi, , Phys. Rev. Lett. 87 (2001) 082001 [hep-ph/0104315]

[10] CMS Collaboration, CMS Physics Analysis Summary, CMS-PAS-SMP-12-012

[11] CMS Collaboration, CMS Physics Analysis Summary, CMS-PAS-FSQ-12-031

[12] CMS Collaboration, CMS Physics Analysis Summary, CMS-PAS-SMP-12-027

[13] G. Sieber, AlphaS extraction and PDF constraints from jet measurements at CMS, http://indico.cern.ch/event/258017/session/1/contribution/42

[14] CMS Collaboration, CMS Physics Analysis Summary, CMS-PAS-SMP-13-002

[15] G. Soyez, A simple description of jet cross-section ratios, Phys. Lett. B 698 (2011) 59 [arXiv:1101:2665]

[16] CMS Collaboration, CMS Physics Analysis Summary, CMS-PAS-SMP-12-022

[17] S. Chatrchyan [CMS Collaboration], Probing color coherence effects in pp collisions at sqrt $(s)=7$ TeV, Eur. Phys. J. C 74 (2014) 2901 [arXiv:1311.5815]

[18] O. Kuprash, Photoproduction of isolated photons, inclusively and with a jet, at HERA, https://indico.cern.ch/event/258017/session/4/contribution/36

[19] ZEUS Collaboration, Further studies of the photoproduction of isolated photons with a jet at HERA, ZEUS preliminary, ZEUS-prel-14-001

H. Abramowicz et al. [ZEUS Collaboration], Further studies of the photoproduction of isolated photons with a jet at HERA, subm. to JHEP (May 2014) [arXiv:1405.7127]

[20] H. Abramowicz et al. [ZEUS Collaboration], Photoproduction of Isolated Photons, Inclusively and with a Jet, at HERA, Phys. Lett. B 730 (2014) 293 [arXiv:1312.1539]

[21] M. Fontannaz, J.P. Guillet and G. Heinrich, Eur. Phys. J. C 21 (2001) 303 [hep-ph/0105121]

M. Fontannaz and G. Heinrich, Eur. Phys. J. C 34 (2004) 191 [hep-ph/03120091]

[22] A.V. Lipatov, M.A. Malyshev and N.P. Zotov, Phys. Rev. D 88 (2013) 074001 [arXiv:1307.3644]

A.V. Lipatov and N.P. Zotov, Phys. Rev. D 81 (2010) 094027 [arXiv:0907.3303]

A.V. Lipatov and N.P. Zotov, Phys. Rev. D 72 (2005) 054002 [hep-ph/0506044] 
[23] J. Cantero Garcia, Measurement of photon and photon+jet production cross sections at $7 \mathrm{TeV}$ and constraints to PDFs, https://indico.cern.ch/event/258017/session/4/contribution/112

[24] G. Aad et al. [ATLAS Collaboration], Measurement of the inclusive isolated prompt photon cross section in pp collisions at $\sqrt{s}=7 \mathrm{TeV}$ with the ATLAS detector using 4.6 fb-1, Phys. Rev. D 89 (2014) 052004 [arXiv:1311.1440]

[25] S. Catani et al., JHEP 0205 (2002) 028, [hep-ph/0204023] P. Aurenche et al., Phys. Rev. D 73 (2006) 094007, [hep-ph/0602133] Z. Belghobsi et al., Phys. Rev. D 79 (2009) 114024, [arXiv:0903.4834]

[26] K. Ocalan, Associated production of a vector boson and jets, https://indico.cern.ch/event/258017/session/4/contribution/44

[27] CMS Collaboration, CMS Physics Analysis Summary, CMS-QCD-11-005, [arXiv:1311.6141].

[28] G. Aad et al. [ATLAS Collaboration], Dynamics of isolated-photon plus jet production in pp collisions at $\sqrt{(s)}=7 \mathrm{TeV}$ with the ATLAS detector, Nucl. Phys. B 875 (2013) 483 [arXiv:1307.6795]

[29] CMS Collaboration, CMS Physics Analysis Summary, CMS-SMP-12-017.

[30] CMS Collaboration, CMS Physics Analysis Summary, CMS-SMP-12-023.

[31] CMS Collaboration, CMS Physics Analysis Summary, CMS-FSQ-12-035.

[32] S. Chatrchyan [CMS Collaboration], Rapidity distributions in exclusive Z+jet and $\gamma+$ jet events in pp collisions at sqrts=7 TeV, Phys. Rev. D 88 (2013) 112009 [arXiv:1310.3082]

[33] CMS Collaboration, CMS Physics Analysis Summary, CMS-SMP-12-004.

[34] J. F. Owens, Rev. Mod. Phys. 59 (1987) 465

[35] M. Sirendi, Associated Z boson production in the forward region, https://indico.cern.ch/event/258017/session/4/contribution/8

[36] R. Aaij et al. [LHCb Collaboration], Study of forward Z+jet production in pp collisions at sâĹŽ=7 TeV, JHEP 01 (2014) 033 [1310:8197]

[37] L. Bellagamba, Production of top pair events with additional radiation using the ATLAS detector at the LHC, https://indico.cern.ch/event/258017/session/4/contribution/115

[38] ATLAS Collaboration, ATLAS PUB Note, ATL-PHYS-PUB-2013-005 (2013).

[39] G. Aad et al. [ATLAS Collaboration], Measurement of ttbar production with a veto on additional central jet activity in pp collisions at sqrt(s)= $7 \mathrm{TeV}$ using the ATLAS detector, Eur. Phys. J. C 72 (2012) 2043 [arXiv:1203.5015]

[40] G. Aad et al. [ATLAS Collaboration], A study of heavy flavor quarks produced in association with top quark pairs at sqrt(s) = 7 TeV using the ATLAS detector, Phys. Rev. D 89 (2014) 072012 [1304.6386]

[41] ATLAS Collaboration, ATLAS PUB Note, ATLAS-CONF-2013-099 (2013).

[42] ATLAS Collaboration, ATLAS-CONF-2011-153 (2011)

[43] ATLAS Collaboration, ATLAS-CONF-2012-126 (2012)

[44] P. M. Szczypka, Measurement of charged particle multiplicities and densities in pp collisions at $7 \mathrm{TeV}$ in the forward region, https://indico.cern.ch/event/258017/session/4/contribution/3

[45] A. Minaenko, Studies of the underlying event with ATLAS, https://indico.cern.ch/event/258017/session/10/contribution/114 
[46] ATLAS Collaboration, ATLAS Note, ATLAS-CONF-2012-164

[47] T. Frueboes, Measurement of the UE activity in pp collisions with the CMS detector, https://indico.cern.ch/event/258017/session/10/contribution/23

[48] CMS Collaboration, CMS Physics Analysis Summary, CMS-PAS-FSQ-12-020.

[49] S. Chatrchyan et al. [CMS Collaboration], Measurement of Strange Particle Production in Underlying Events in proton-proton collisions at âLĽ̌s = 7 TeV, Phys. Rev. D 88 (2013) 052001 [arXiv:1305.6016]

[50] S. Chatrchyan et al. [CMS Collaboration], Jet and underlying event properties as a function of particle multiplicity in proton-proton collisions at sqrt(s) = 7 TeV, Eur. Phys. J. C 73 (2013) 2674 [arXiv:1310.4554]

[51] CMS Collaboration, CMS Physics Analysis Summary, CMS PAS TOP-13-007

[52] P. Gunnellini, UE event tunes and double parton scattering/Measurement of four-jet production in proton-proton collisions at $\operatorname{sqrt}(\mathrm{s})=7 \mathrm{TeV}$, https://indico.cern.ch/event/258017/session/10/contribution/264

[53] CMS Collaboration, CMS Physics Analysis Summary, CMS-PAS-GEN-14-001

[54] A. Grebenyuk, Measurement of double-parton interactions in W+2 jets events with the CMS detector, https://indico.cern.ch/event/258017/session/10/contribution/24

[55] S. Chatrchyan [CMS Collaboration], Study of double parton scattering using $W+2$-jet events in proton-proton collisions at sqrt(s) = $7 \mathrm{TeV}$, JHEP 1403 (2014) 032 [arXiv:1312.5729]

[56] S. Chatrchyan [CMS Collaboration], Measurement of four-jet production in proton-proton collisions at $\operatorname{sqrt}(s)=7 \mathrm{TeV}$, Phys. Rev. D 89 (2014) 092010 [arXiv:1312.6440]

[57] H. Arnold, First measurement of associated vector boson plus prompt charmonium production at the ATLAS experiment, https://indico.cern.ch/event/258017/session/4/contribution/128

[58] G. Aad et al. [ATLAS Collaboration], Measurement of the production cross section of prompt J/Psi mesons in association with a $W$ boson in pp collisions at sqrt(s)= $7 \mathrm{TeV}$ with the ATLAS detector, JHEP 1404 (2014) 172 [arXiv:1401.2831]

[59] R. Aaij et al. [LHCb Collaboration], Observation of associated production of a Z boson with a D meson in the forward region, JHEP 04 (2014) 091 [1401:3245]

[60] A. Szczurek, Double charmed meson production at the LHC: Single-versus double-parton scattering mechanism, https://indico.cern.ch/event/258017/session/4/contribution/193

[61] H. Martinez Bruzual, Measurement of the transverse momentum of the Z boson and NLO MC tuning, https://indico.cern.ch/event/258017/session/4/contribution/110

[62] G. Aad et al. [ATLAS Collaboration], Measurement of the $Z / \gamma^{*}$ boson transverse momentum distribution in pp collisions at $\sqrt{s}=7 \mathrm{TeV}$ with the ATLAS detector, CERN-PH-EP-2014-075 (2014) [arXiv:1406.3660]

[63] A. Banfi et al., Optimisation of variables for studying dilepton transverse momentum distributions at hadron colliders, Eur. Phys. J. C 71 (2011) 1600 [arXiv:1009.1580]

[64] P. Sopicki, Search for QCD Instantons using the H1 experiment at HERA, https://indico.cern.ch/event/258017/session/4/contribution/88

[65] H1 Collaboration, H1 preliminary, H1prelim-14-031 
[66] M. Gibbs et al., Conf. Proc. C950424 (1995) 341 [hep-ph/9506392]

A. Ringwald and F. Schrempp, Comp. Phys. Comm. 132 (2000) 267 [hep-ph/9911516]

[67] J. Olsson, Analysis of Feynman Scaling in Photon and Neutron Production in the Very Forward Direction in Deep-Inelastic Scattering at HERA, https://indico.cern.ch/event/258017/session/4/contribution/83

[68] V. Andreev et al. [H1 Collaboration], Measurement of Feynman-x Spectra of Photons and Neutrons in the Very Forward Direction in Deep-Inelastic Scattering at HERA, Eur. Phys. J. C 74 (2014) 2915 [arxiv:1404.0201]

[69] G. Cibinetto, Light meson production in e+e-annihilation at BABAR, https://indico.cern.ch/event/258017/session/4/contribution/190

[70] J. P. Lees et al. [BABAR Collaboration], Precise Measurement of the e+e- $->$ pi+ pi- (gamma) Cross Section with the Initial-State Radiation Method at BABAR, Phys. Rev. D 86 (2012) 032013 [arXiv:1205.2228]

[71] B. Aubert et al. [BABAR Collaboration], Precise measurement of the e+e-to pi+ pi- (gamma) cross section with the Initial State Radiation method at BABAR, Phys. Rev. Lett. 103 (2009) 231801 [arXiv:0908.3589]

[72] J. P. Lees et al. [BABAR Collaboration], Precision Measurement of the e+e- $->K+K$-(gamma) Cross Section with the Initial-State Radiation Method at BaBar, Phys. Rev. D 88 (2013) 032013 [arXiv:1306.3600]

[73] M. Misheva, Detailed study of the Ke4 decay mode properties, https://indico.cern.ch/event/258017/session/4/contribution/177

[74] J.R. Batley [NA48/2 Collaboration], New measurement of the charged kaon semileptonic (Ke4) decay Branching Ratio and Hadronic Form Factors, Phys. Lett. B 715 (2012) 105 [arXiv:1206.7065]

[75] NA48/2 Collaboration, preliminary result, 2014

[76] P. Petrov, Measurement of the rare decay $K^{ \pm} \rightarrow \pi^{ \pm}$gamma gamma, https://indico.cern.ch/event/258017/session/4/contribution/176

[77] C.Lazzeroni et al. [NA62 Collaboration], Study of the K+- to pi+- gamma gamma decay by the NA62 experiment, Phys. Lett. B 732 (2014) 65 [arXiv:1402.4334]

[78] J. R. Batley et al. [NA48/2 Collaboration], A new measurement of the K+- to pi+- gamma gamma decay at the NA48/2 experiment, Phys. Lett. B 730 (2014) 141 [arXiv:1310.5499]

[79] R. Maciula, Production of dijets with large rapidity separation: Mueller-Navalet mechanism versus double-parton scattering, https://indico.cern.ch/event/258017/session/10/contribution/191

[80] B. Ducloue, Confronting BFKL dynamics with experimental studies of Mueller-Navelet jets at the LHC, https://indico.cern.ch/event/258017/session/10/contribution/242

[81] H. Paukkunen, LHC data challenges the contemporary parton-to-hadron fragmentation functions, https://indico.cern.ch/event/258017/session/4/contribution/2

[82] M. Jack, High Energy Jets at the LHC, https://indico.cern.ch/event/258017/session/4/contribution/269

[83] H. Van Haevermaet, Higgs boson as a gluon trigger, https://indico.cern.ch/event/258017/session/4/contribution/122

[84] K. Kutak, Azimuthal decorelations in forward central dijets production at LHC within high energy actorization, https://indico.cern.ch/event/258017/session/4/contribution/124 


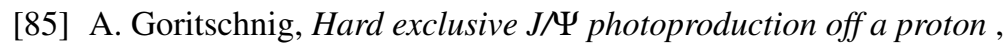
https://indico.cern.ch/event/258017/session/4/contribution/241

[86] J.-P. Lansberg, Associated-quarkonium production, https://indico.cern.ch/event/258017/session/4/contribution/18

[87] P. Lebiedowicz, Applications of the tensor pomeron model to exclusive central diffractive meson production, https://indico.cern.ch/event/258017/session/10/contribution/194 